\title{
A Novel Dynamic Measurement System for Evaluating the Braking Coordination of Articulated Vehicles
}

\author{
Xu Li, Wei Chen, and Qimin Xu \\ School of Instrument Science and Engineering, Southeast University, Nanjing 210096, China \\ Correspondence should be addressed to Xu Li; lixu.mail@163.com
}

Received 14 May 2016; Revised 6 June 2016; Accepted 20 June 2016

Academic Editor: Stephane Evoy

Copyright (C) $2016 \mathrm{Xu} \mathrm{Li} \mathrm{et} \mathrm{al.} \mathrm{This} \mathrm{is} \mathrm{an} \mathrm{open} \mathrm{access} \mathrm{article} \mathrm{distributed} \mathrm{under} \mathrm{the} \mathrm{Creative} \mathrm{Commons} \mathrm{Attribution} \mathrm{License,} \mathrm{which}$ permits unrestricted use, distribution, and reproduction in any medium, provided the original work is properly cited.

\begin{abstract}
The braking coordination between tractor and semitrailer is vital to the safety of articulated vehicles. Traditional evaluation about braking coordination is based on the pressure measurement along air braking pipeline, which needs to change original braking structure to install gauges and cannot directly reflect the final braking coordination of different wheels. To overcome these limitations, this paper proposes a novel dynamic measurement system for evaluating the braking coordination of articulated vehicles. During the brake test, all wheel velocities of the whole articulated vehicle are synchronously obtained through a specially designed distributed acquisition platform. To effectively eliminate gross errors and noises in wheel angular velocity data, a 3order autoregressive (AR) model and an improved-thresholding wavelet filtering algorithm are developed. Further, a novel direct evaluation method about braking coordination is proposed according to the differences in angular velocity dropping time of all wheels. Finally, the overall system is assessed through real field tests. The results validate the feasibility and effectiveness of the proposed system.
\end{abstract}

\section{Introduction}

To reduce the accidents of tractor-semitrailers and improve their safety, many efforts have been dedicated to investigating active safety systems including antilock braking system or electronic stability control [1-3], developing driver assistance systems [4], improving the design of braking system [5], and so forth. From the view of actual applications, when such systems have been introduced into tractor-semitrailers, it is also important to carry out proper performance measurements to evaluate their effect on the safety. Through effective performance assessment, the assessment results can help government management sectors to establish proper access and retreat mechanism about articulated vehicles. Besides, the performance assessment gives both truck manufacturers and operators more flexibility and opportunities for innovation in their continuing efforts to improve safety while boosting productivity and lowering costs.

Undisputedly, among different performance evaluations, the braking performance is primary and vital. The world's major economies have issued the standards/rules which hold legal demands regarding braking tests and performance of braking systems, for example, FMVSS 105/121/135 in USA [6], ECE R13 in the European Commission [7], and GB7258/ 26778/12676 in China [8-10]. Generally, braking tests are performed on certain road-friction surface with a preset initial velocity, and the quantities such as the stopping distance and the mean fully developed deceleration (MFDD) act as evaluation parameters. Although these evaluation parameters can accurately reflect the braking performance of individual vehicles, they are insufficient to assess the braking performance of articulated vehicles.

The articulated vehicle is a combination of tractor and semitrailer, which are connected with each other through the fifth wheel (kingpin). If the braking system of tractor is not coordinated with that of semitrailer, it may cause severe accidents such as jackknife, snaking, and trailer-swing, which are unique to articulated vehicles [11, 12]. The braking coordination of articulated vehicle means that the maximal difference of braking delay time between any two wheel axles of the whole articulated vehicle should be small enough to ensure the consistency and stability in actual braking conditions. 


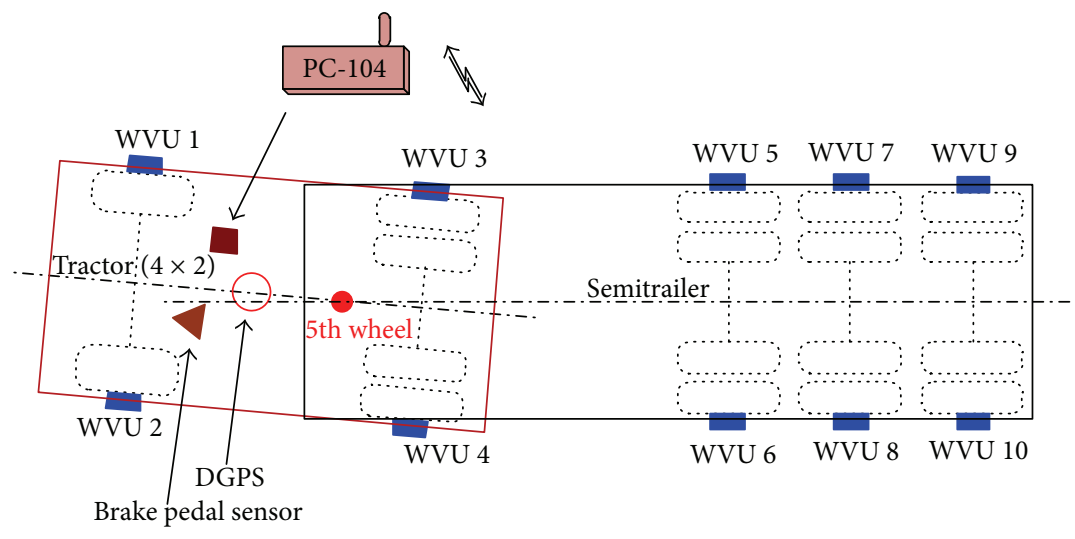

FIGURE 1: Overall configuration of the proposed system.

Traditionally, the braking coordination is evaluated through analyzing the differences in the pressure build-up time of different wheel axles [13-15]. For this kind of evaluation, pressure gauges need to be installed along air braking pipeline to measure brake pressures at different wheel axles over time. However, this kind of evaluation has several limitations in actual applications. First, it will take much time to install and disassemble pressure gauges during the whole test, which is especially inconvenient for certain-scale tests. Besides, the installation of pressure gauges will change original braking structure and may lead to potential unsafe impacts. Moreover, since this kind of evaluation is performed based on the differences in the pressure build-up time of different wheel axles rather than the velocity dropping time (i.e., braking) of different wheels under actual braking situation, it cannot directly provide the final braking coordination result from the view of kinematics.

In this paper, a novel dynamic measurement system for evaluating the braking coordination of articulated vehicles is proposed. It evaluates the braking coordination performance through analyzing the differences in the angular velocity dropping time of all wheels during the dynamic brake of articulated vehicles, which is executed on actual road surfaces. The novel aspects of the proposed system can be summarized as follows. (1) It can directly evaluate the final braking coordination of articulated vehicles because it is executed in real dynamic brake situation and directly measures the differences in the angular velocity dropping time of all wheels. (2) It does not need to change original braking pipeline of tractorsemitrailers and has the advantages of easiness to install, convenient maintenance, low cost, and so forth.

Section 2 gives the overview of the overall system and describes the developed wheel velocity unit in particular. Section 3 presents data processing algorithms. The evaluation method about the braking coordination is proposed in Section 4. Then, field test results are provided in Section 5. Finally, the conclusion is given in Section 6.

\section{The Proposed Measurement System}

2.1. Overview of Overall System. The proposed system consists of an embedded main computer, a brake pedal sensor, a differential global positioning system (DGPS), and a group of wheel velocity units (WVUs). The overall configuration is shown in Figure 1. The main computer is developed based on PC-104 bus with stack structure, which is characterized by low consumption and small size as well as high reliability and thus is very suitable for vehicular environments. The brake pedal sensor is utilized to determine the brake beginning time, that is, reference time 0 of braking, while DGPS is integrated to measure the velocity of the tractor. The WVU is specially designed to measure and store angular velocity of each wheel, which is installed on the wheel hub through the fixture specially designed, and it exchanges data with the main computer through wireless communication.

To realize effective and efficient braking coordination test, the working mechanism of the overall system follows the idea of "centralized control, distributed collection, and postprocessing." According to this idea, we develop the software on the main computer to control the whole system. Besides, the software collects wheel velocity data based on multithread technique and processes all the data. Specifically, at the beginning of brake coordination test, the centralized-control main computer triggers all WVUs to work as well as the inner AD acquisition card to collect the pedal sensor data. Simultaneously, it starts to collect the velocity data of the tractor through DGPS. During the test, the angular velocity of each wheel is measured and stored by the corresponding WVU. Once the test is finished, the main computer acquires the wheel angular velocity information of each WVU through wireless communication. Finally, data processing and braking coordination evaluation are performed on the main computer.

2.2. Design of $W V U$. As more and more articulated vehicles are equipped with active safety systems, it seems easy and convenient to obtain wheel velocity information through the Controller Area Network (CAN) bus. However, for such information, one major problem is that the resolution is low, usually one pulse per revolution. Thus, the measurement accuracy cannot be ensured when the velocity becomes low. To realize reliable evaluation of braking coordination of articulated vehicles, we specially design the WVU module to measure the wheel velocity accurately. 


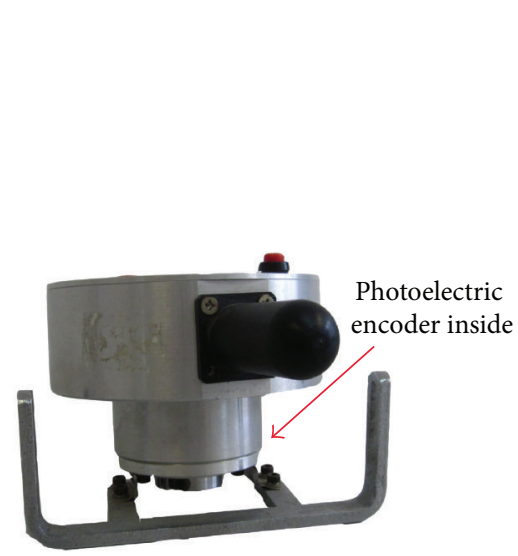

(a)

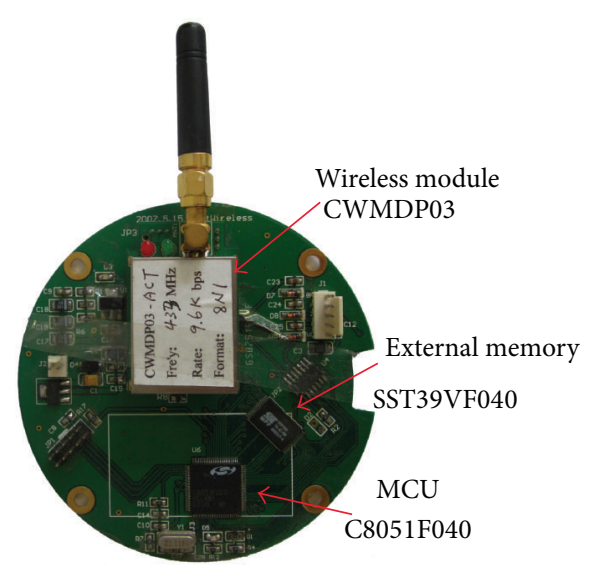

(b)

Figure 2: Developed WVU module. The complete module (a) and the PCB (b).

Figure 2 illustrates the developed WVU module. It is mainly composed of four parts, that is, photoelectric encoder, microcontrol unit (MCU), external memory, and wireless module. The photoelectric encoder is adopted to measure wheel velocity, which has stronger anti-interference ability over the devices based on electromagnetic induction or Hall Effect. In actual applications, the photoelectric encoder is installed to be coaxial with the wheel, which outputs the pulse sequence as the wheel rotates. To achieve high accuracy, we adopt the photoelectric encoder with the resolution of 1024 . The pulses output by the photoelectric encoder are fed to MCU after proper conditioning. The C8051F040, produced by Silicon Lab, is chosen as MCU in view of its low consumption, high reliability, and full integration including input/output port, Universal Asynchronous Receiver/ Transmitter (UART), Programmable Counter Array (PCA), and CAN controller. Besides, the external memory utilizing SST39VF040 with $512 \mathrm{~KB}$ is extended in WVU to ensure enough storage space. The wireless module CWMDP03 using $433 \mathrm{MHz}$ communication frequency is utilized to transfer the information between WVU and the main computer including control commands and angular velocity data. This wireless module has obvious advantages in the penetrability and propagation distance, which is quite suitable for test scenarios of articulated vehicles.

To measure angular velocity, there are two commonly used methods, that is, frequency measurement and period measurement, respectively. The former is more accurate at high velocity, while the latter is more accurate at low velocity. To take advantage of the two methods and adapt to a wide range of velocities, we adopt a combined method. That is, when the wheel velocity is higher than a certain threshold, the frequency measurement method is utilized. On the contrary, if the velocity is lower than this threshold, the period measurement method is adopted. Through such processing, the measured angular velocity data can achieve high measurement accuracy.

\section{Data Processing Algorithm}

To effectively evaluate the braking coordination, it is vital to obtain accurate angular velocity data of all wheels. However, during emergent brake test, it will inevitably cause measurement noises and even gross errors at the beginning of brake due to fluctuations of braking air pressure, vibrations of vehicle body, imperfections of fixture, changes of tire-road friction conditions, and so forth. To eliminate these adverse effects, a data processing algorithm based on time series analysis is first proposed to detect and correct the potential gross errors. Then, an improved-thresholding wavelet filtering algorithm is developed to further remove the noises in the wheel angular velocity measurements.

3.1. Detecting and Correcting Gross Errors Based on $A R$ Model. Due to the advantages of low computational burden, good real-time performance, and high prediction accuracy, autoregressive (AR) model is one of the most popular models in time series analysis and has been widely used in practical applications, such as traffic flow forecast and gyroscope drift modeling [16-18].

Traditional methods such as $3 \delta$-criterion, Grubbs criterion, Chauvenet criterion, and Dixon criterion [19-22] are mainly utilizing simple statistics to identify gross errors, which do not consider time series features of data and often cannot obtain satisfactory results when there exist consecutive gross errors. Unlike them, AR model is based on mathematical modeling of a group of time series data assuming that each value of the series is dependent on a weighted sum of the "previous" values of the same series. The AR model can be described as follows:

$$
y(\phi)=-\sum_{n=1}^{p} a_{n} y(\phi-n)+\varepsilon(\phi),
$$

where $p$ is the order of AR model, $\phi$ is the time stamp, $a_{n}$ $(n=1,2, \ldots, p)$ are the AR model parameters, and $\varepsilon(\phi)$ is a purely random process with variance $\theta^{2}$. 
For AR model, the estimation of AR model parameters $a_{n}$ $(n=1,2, \ldots, p)$ is the main consideration. Several methods, including Yule-Walker, covariance, and Burg's methods, have been presented to estimate AR model parameters in the literature [23]. Compared with the Yule-Walker and covariance methods, Burg's method can minimize both forward and backward prediction errors in the least square sense and provide more accurate estimates of AR parameters even for short data records.

Obviously, the wheel angular velocity data can be regarded as a sequence of data points indexed by time. From the point of time series, there is a certain temporal correlation among consecutive wheel angular velocity data in a long enough time interval. Taking this point into consideration, current wheel angular velocity data may have some relationship with past wheel angular velocity data. Therefore, the wheel angular velocity data can be modeled as AR process.

Assuming $x(t)(t=1,2, \ldots, k, k>200)$ is the wheel angular velocity data of one axle, the first 200 pieces of data, namely, $x(l)(l=1,2, \ldots, 200)$, are chosen as samples to complete the modeling in this study. To establish AR model for $x(l)$, they should be first processed by differentiation procedure to remove nonstationarity owing to an assumption for time series modeling that the data series should be stationary.

After stationary processing, the next step is to determine the order of AR model. To identify the AR model order, the autocorrelation $\left(\mathrm{ACF}, \widehat{\rho}_{\xi}\right.$ ) and partial autocorrelation (PACF, $\widehat{\varphi}_{\xi \xi}$ ) of stationary wheel angular velocity data are first computed as follows:

$$
\begin{aligned}
& \widehat{\rho}_{\xi}=\frac{\widehat{r}_{\xi}}{\widehat{r}_{0}}, \\
& \widehat{r}_{0}=\frac{1}{200} \sum_{q=1}^{200}[x(q)]^{2}, \\
& \widehat{r}_{\xi}=\frac{1}{200} \sum_{q=1}^{200-\xi} x(q) \cdot x(\xi+q), \\
& \left(\begin{array}{c}
\widehat{\varphi}_{\xi 1} \\
\widehat{\varphi}_{\xi 2} \\
\vdots \\
\widehat{\varphi}_{\xi \xi}
\end{array}\right) \\
& =\left(\begin{array}{ccccc}
1 & \hat{\rho}_{1} & \hat{\rho}_{2} & \cdots & \hat{\rho}_{\xi-1} \\
\hat{\rho}_{1} & 1 & \cdots & \cdots & \cdots \\
\vdots & \vdots & \vdots & \vdots & \vdots \\
\cdots & \cdots & \cdots & \cdots & \hat{\rho}_{1} \\
\hat{\rho}_{\xi-1} & \cdots & \hat{\rho}_{2} & \hat{\rho}_{1} & 1
\end{array}\right)^{-1}\left(\begin{array}{c}
\hat{\rho}_{1} \\
\hat{\rho}_{2} \\
\vdots \\
\hat{\rho}_{\xi}
\end{array}\right),
\end{aligned}
$$

where $\xi=1,2, \ldots, 199$ and $q$ is a positive integer.

Then, according to the judge rule based on ACF and PACF, the order of AR model can be determined. If PACF cuts off to zero after lag $p$ and the exponential or sinusoidal
ACF decays to zero, $p$ is selected as the order of AR model. Utilizing this rule, it can be determined that the order $p$ is 3 . That is, the AR(3) model is established to model the wheel angular velocity data.

When the order of AR model has been determined, the model parameters can be estimated. Because the length of $x(l)(l=1,2, \ldots, 200)$ is 200 , it is a short data record from the perspective of time series analysis. To achieve more accurate estimation of model parameters, Burg's method is chosen in this study. Here, we define the forward prediction error $e_{f_{n}}(\phi)$ and backward prediction error $e_{b_{n}}(\phi)$ in a pair of orderrecursive equations:

$$
\begin{aligned}
& e_{f_{n}}(\phi)=e_{f_{n-1}}(\phi)+r_{n} e_{b_{n-1}}(\phi-1), \\
& e_{b_{n}}(\phi)=e_{b_{n-1}}(\phi-1)+r_{n} e_{f_{n-1}}(\phi),
\end{aligned}
$$

where $(n+1) \leq \phi \leq 200,1 \leq n \leq 3$, and $r_{n}$ is the reflection coefficient and is defined as

$$
r_{n}=\frac{-2 \sum_{\phi=n+1}^{200}\left[e_{f_{n-1}}(\phi) \cdot e_{b_{n-1}}(\phi-1)\right]}{\sum_{\phi=n+1}^{200} e_{f_{n-1}}^{2}(\phi)+\sum_{\phi=n+1}^{200} e_{b_{n-1}}^{2}(\phi-1)} .
$$

Further, we can estimate the AR(3) model parameters according to the following five steps.

Step 1 (initialization). The initial condition is $e_{f_{0}}(\phi)=$ $e_{b_{0}}(\phi)=x(\phi)$. The initial value of $\theta^{2}$ is calculated as

$$
\theta_{0}^{2}=\frac{1}{200} \sum_{\phi=1}^{200} x^{2}(\phi)
$$

Then, set $n=1$ and go to the second step.

Step 2 (calculate the reflection coefficients $r_{n}$ and $\theta_{n}^{2}$ ). $r_{n}$ can be obtained using (4), while $\theta_{n}^{2}$ is given as

$$
\theta_{n}^{2}=\left(1-r_{n}^{2}\right) \theta_{n-1}^{2}
$$

Step 3 (calculate the parameters $\left.a_{n, i}(i=1,2, \ldots, n)\right)$. The parameters are calculated as

$$
\begin{aligned}
& a_{n, n}=r_{n}, \\
& a_{n, i}=a_{n-1, i}+a_{n, n} a_{n-1, n-i} .
\end{aligned}
$$

Step 4. Update $e_{f_{n}}(\phi)$ and $e_{b_{n}}(\phi)$ employing (3).

Step 5. If $n$ is equal to 3 , the process is ended. Otherwise, set $n=n+1$ and jump to Step 2 .

When the five steps above are completed, the $\operatorname{AR}(3)$ model parameters and the variance of purely random process can be estimated as follows:

$$
\begin{aligned}
& a_{1}=a_{3,1}, \\
& a_{2}=a_{3,2}, \\
& a_{3}=a_{3,3}, \\
& \theta=\theta_{3} .
\end{aligned}
$$




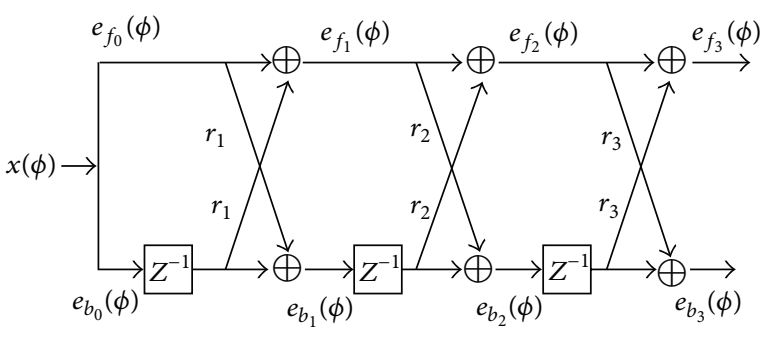

FIgURE 3: Burg's lattice filter for parameters estimation of $\operatorname{AR}(3)$ model $\left(Z^{-1}\right.$ : time delay).

For further clarification, the recursive structure of parameter estimation process can be described by Burg's lattice filter, which is shown in Figure 3.

Once the process of modeling the wheel angular velocity data is completed, the developed AR(3) model can be utilized to detect gross errors of wheel angular velocity. Initially, we assume $\widehat{x}(t)$ as the prediction of $x(t)$ and set $\widehat{x}(198)=x(198)$, $\widehat{x}(199)=x(199)$, and $\widehat{x}(200)=x(200)$. From $t=201$, $x(t-1), x(t-2)$, and $x(t-3)$ are extracted from $x(t)(t=$ $1,2, \ldots, k, k>200)$ in order every time. According to $\mathrm{AR}(3)$ model, $\widehat{x}(t)$ can be estimated as

$$
\widehat{x}(t)=a_{1} x(t-1)+a_{2} x(t-2)+a_{3} x(t-3) .
$$

If $|\widehat{x}(t)-x(t)|$ is more than a proper threshold, $x(t)$ is identified as a gross error and replaced by $\widehat{x}(t)$.

3.2. Eliminating Noises Based on Wavelet Transform. Applying the proposed AR(3) model, we can effectively detect and repair gross errors caused in emergency brake situations. However, the remaining noises still make a huge difficulty to accurately determine the braking time differences between different wheels. To further improve the accuracy of wheel angular velocity data, an improved wavelet filtering algorithm is designed to remove the noises and increase the signal-tonoise ratio of wheel angular velocity data.

As a useful mathematical tool, wavelet transform has quickly spread to a whole spectrum of applications in science and engineering fields in a relatively brief period [24-26]. Due to the powerful facilities of wavelet, it is capable of denoising a signal without introducing appreciable degradation of the original signal.

To achieve wavelet filtering processing, a suitable wavelet should be selected first to perform discrete wavelet transform (DWT) of wheel angular velocity signal. To obtain both good decomposition and reconstruction of wheel angular velocity signal, the selected wavelet should be orthogonal. Moreover, the selected wavelet should have compact support and highorder vanishing moment, which is the benefit of detecting singular point in wheel angular velocity signal. In addition, a wavelet with moderate supporting length is preferred so as to reduce the computational burden. In this paper, the "Daubechies5" wavelet in the Daubechies family is chosen to perform DWT.

In addition to wavelet function, the number of the levels of decomposition (LOD) for DWT also has a significant influence on the filter performance. In the vehicle application, an appropriate LOD can be selected when the noises are removed and all true motion dynamics components of the vehicle are preserved in the filtered signal. Unfortunately, there is no coherent methodology to determine how many levels of decomposition should be used at present. In this paper, a proper LOD is achieved by analyzing the frequency band property after DWT. According to DWT, each level of decomposition divides the spectrum of the original signal into different subbands. If one LOD is applied for the wheel angular velocity signal with sampling frequency $f_{s}=100 \mathrm{~Hz}$, we will have a spectrum in the range $0 \sim 25 \mathrm{~Hz}$ for approximation coefficients and a spectrum in the range $25 \sim 50 \mathrm{~Hz}$ for detail coefficients. Decomposing the wheel angular velocity signal iteratively, we can further gain the frequency band of each approximation part and each detail part.

Since the fundamental vehicle dynamics are generally below $2 \sim 3 \mathrm{~Hz}$, these motion dynamics are preserved in lowfrequencies components (approximation parts) after DWT. After applying four LOD, the output band of approximation coefficients is limited to $3.125 \mathrm{~Hz}$, which nearly reaches $3 \mathrm{~Hz}$. Therefore, using four LOD is proper when taking into account the fact that a sampling frequency of $f_{s}=100 \mathrm{~Hz}$ is used for the wheel angular velocity signal.

Moreover, the selection of an appropriate threshold is another crucial task as it directly affects the denoising results. In current study, the threshold is selected based on the heuristics variant of Stein's Unbiased Risk Estimate (SURE), which is a conservative method and can avoid the loss of wavelet coefficients associated with vehicle motion. The calculation steps of the threshold $\mathrm{Th}_{m}$ at level of $m$ are designed as follows.

Step 1 (calculating the noise intensity of signal). The noise intensity of wheel angular velocity signal at level of $m$ is expressed as

$$
\sigma_{m}=\frac{\operatorname{median}\left(\left|D_{m, j}\right|\right)}{0.6745},
$$

where $\sigma_{m}$ is the noise intensity, $D_{m, j}$ represents detail coefficient and is the $j$ th element of detail part $D_{m}, \operatorname{median}\left(\left|D_{m, j}\right|\right)$ denotes the median of $\left|D_{m, j}\right|\left(j=1,2, \ldots, N_{m}\right)$, and $N_{m}$ is the length of $D_{m}$.

Step 2 (calculating the universal threshold). According to the method proposed by Donoho and Johnstone [27], the universal threshold $\mathrm{Th}_{1, m}$ at level of $m$ can be computed as

$$
\mathrm{Th}_{1, m}=\sigma_{m} \sqrt{2 \ln \left(N_{m}\right)} .
$$

Step 3 (calculating Stein's unbiased likelihood threshold). Through computing the square of detail coefficients at $m$ level and sorting them from the smallest to the largest [28], we can obtain

$$
\begin{aligned}
& \boldsymbol{\omega}=\left[\omega_{1}, \omega_{2}, \ldots, \omega_{j}, \ldots, \omega_{N_{m}}\right], \\
& \mathbf{R}=\left[R_{1}, R_{2}, \ldots, R_{j}, \ldots, R_{N_{m}}\right],
\end{aligned}
$$


where $\omega_{j}=\left(D_{m, r}\right)^{2}\left(r=1,2, \ldots N_{m}\right), \omega_{1} \leq \omega_{2} \leq \cdots \leq \omega_{N_{m}}$, and $R_{j}=\left(N_{m}-2 j+\left(N_{m}-j\right) \omega_{i}+\sum_{\gamma=1}^{N_{m}} \omega_{\gamma}\right) / N_{m}$. If the minimum element in $\mathbf{R}$ is $R_{b}$, the $b$ th element $\omega_{b}$ of $\boldsymbol{\omega}$ is selected, where $b$ is an integer and $1 \leq b \leq N_{m}$. Then, Stein's unbiased likelihood threshold $\mathrm{Th}_{2, m}$ at level of $m$ can be ascertained as follows:

$$
\mathrm{Th}_{2, m}=\sigma_{m}\left(\omega_{b}\right)^{1 / 2} .
$$

Step 4 (calculating heuristic Stein's unbiased likelihood threshold). The final heuristic Stein unbiased likelihood threshold $\mathrm{Th}_{m}$ at $m$ level can be determined as

$$
\mathrm{Th}_{m}= \begin{cases}\mathrm{Th}_{1, m}, & \eta<\mu, \\ \min \left(\mathrm{Th}_{1, m}, \mathrm{Th}_{2, m}\right), & \eta \geq \mu\end{cases}
$$

where $\eta=\left(H_{w}-N_{m}\right) / N_{m}, H_{w}=\sum_{j=1}^{N_{m}} \omega_{j}$, and $\mu=$ $\left(\log _{2} N_{m}\right)^{3 / 2} / \sqrt{N_{m}}$.

After obtaining the suitable threshold, we can adopt shrinkage scheme to perform thresholding on detail coefficients to suppress or remove the noise component. Softthresholding is the most commonly used shrinkage scheme for threshold denoising because hard-thresholding causes discontinuity in the thresholded coefficients. However, softthresholding generates biased outputs, which may result in additional error sources in certain conditions. To overcome this limitation, a new improved-thresholding technique is proposed:

$$
\widetilde{D}_{m, j}= \begin{cases}\operatorname{eta}\left(D_{m, j} ; \mathrm{Th}_{m}\right), & \left|D_{m, j}\right| \geq \mathrm{Th}_{m}, \\ 0, & \left|D_{m, j}\right|<\mathrm{Th}_{m},\end{cases}
$$

where $\widetilde{D}_{m, j}$ is the detail coefficient processed by thresholding and function eta $(\cdot)$ is defined as

$$
\begin{aligned}
\operatorname{eta} & (Y ; X) \\
= & \operatorname{sgn}(Y)(|Y|-X) \\
& -\left\{\frac{2}{1+\exp [\operatorname{sgn}(Y)(|Y|-X) / 2 X]}-1\right\} X,
\end{aligned}
$$

where $\operatorname{sgn}(\cdot)$ is the $\operatorname{symbol}$ function and $\exp (\cdot)$ is the exponential function.

The proposed thresholding scheme above is a more continuous approach which preserves the highest amplitude coefficients and has smooth transition from noisy to important coefficients. Thus, it can help keep the continuity and reduce the impact of a biased signal.

To provide a more clear description, the improvedthresholding wavelet filtering algorithm developed above is summarized briefly as follows.

(1) Decomposition. Selecting "Daubechies5" as the wavelet function, we perform four levels of decomposition on the wheel angular velocity data using DWT.

(2) Threshold Processing. First, the suitable threshold is determined by (14) based on the heuristics variant of SURE.
Then, all detail coefficients are processed by the improvedthresholding scheme according to (15).

(3) Reconstruction. Through the inverse DWT, the denoised wheel angular velocity data can be obtained using the approximation coefficients at the level of four and the thresholded detail coefficients from levels of one to four.

\section{Evaluation Method about the Braking Coordination}

Utilizing all wheel angular velocity data processed by the algorithms presented above, we can carry out the braking coordination evaluation under unified time frame.

During braking coordination test, the brake beginning time of the whole articulated vehicle, which is represented by $t_{b}$ here, can be easily determined according to the brake pedal signal. Obviously, due to the delay of air braking pipeline, the time when each wheel begins to decelerate is lagging behind $t_{b}$. Theoretically, if we can determine the beginning time of rapid angular velocity decrease of each wheel, which is denoted by $t_{i}^{j}$, we can use the following difference to describe the braking delay time of each wheel relative to $t_{b}$ :

$$
T_{i}^{j}=t_{i}^{j}-t_{b}
$$

where the superscript $j=l$ or $j=r$ represents the left or right wheel, respectively, while the subscript $i\left(i=1, \ldots, N_{a}\right)$ denotes the $i$ th axle of articulated vehicle which is assumed to have $N_{a}$ wheel axles. Further, the braking coordination can be directly evaluated through analyzing the differences of the braking delay time of different wheels.

However, it is very difficult to accurately determine the time $t_{i}^{j}\left(j=l, r\right.$ and $\left.i=1, \ldots, N_{a}\right)$ in practice. Due to the influences of nonlinear factors such as uncertain air braking delay and fluctuation and vehicle vibrations, there does not exist an obvious and certain time which can accurately represent the beginning of rapid angular velocity decrease of any wheel. Figure 4 shows the actual angular velocity of one wheel (after data processing) and braking pedal force during one braking coordination dynamic test. In Figure $4, \omega_{\max }$ and $F_{\text {max }}$ denote the maximal wheel angular velocity before the brake and the maximal brake pedal force, respectively.

From Figure 4, although it is difficult to determine $t_{i}^{j}$, the wheel angular velocity will follow a monotonous and stable dropping trend during an interval after the beginning phase of braking process. For example, during the interval between $0.7 \omega_{\max }$ and $0.3 \omega_{\max }$, the wheel angular velocity experiences a nearly constant deceleration process. Based on this regular trend, a novel method for evaluating the braking coordination performance of articulated vehicle is proposed, which includes three steps as follows.

Step 1. Ascertain the brake beginning time of the whole articulated vehicle, that is, $t_{b}$ : select the time when the brake pedal force increases to $0.5 F_{\max }$ as $t_{b}$.

Step 2. Determine the average braking delay time (ABDT) of all wheel axles. 


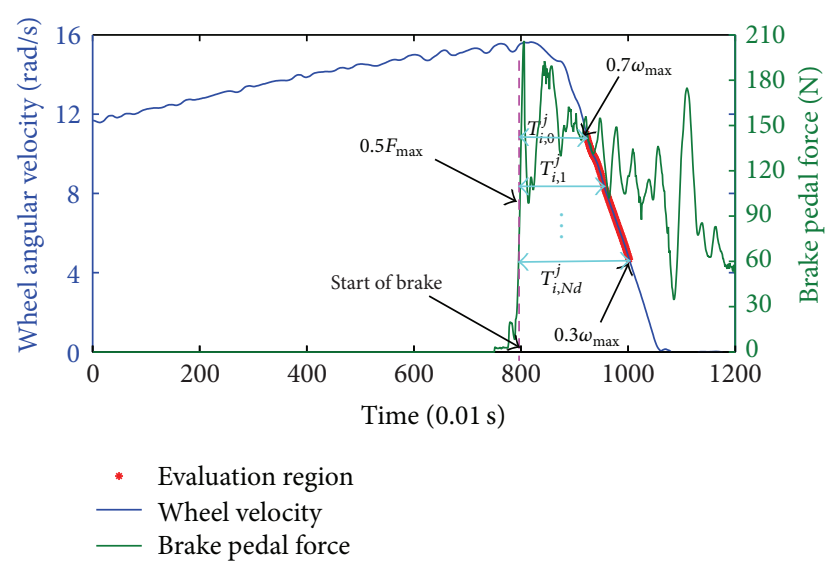

Figure 4: Angular velocity of one wheel and braking pedal force during one braking coordination test.

First, the angular velocity region from $0.7 \omega_{\max }$ to $0.3 \omega_{\max }$ is selected as the evaluation region and is equally divided into $N_{d}$ parts. Then, according to Figure 4 , we can determine all the differences $T_{i, q}^{j}\left(q=0,1,2, \ldots, N_{d}\right)$ between the time when the wheel angular velocity decreases to $(0.7-0.4$. $\left.q / N_{d}\right) \omega_{\max }\left(q=0,1,2, \ldots, N_{d}\right)$ and the time $t_{b}$. Further, the average braking delay time (ABDT) of any individual wheel is defined as

$$
\bar{T}_{i}^{j}=\frac{1}{\left(N_{d}+1\right)} \sum_{q=0}^{N_{d}} T_{i, q}^{j} \quad\left(j=l, r, i=1, \ldots, N_{a}\right) .
$$

Besides, considering that the left and right wheels on the same axle almost have the same average braking delay time in practice, we can further define the ABDT of the $i$ th wheel axle as

$$
\bar{T}_{i}=\frac{1}{2}\left(\bar{T}_{i}^{l}+\bar{T}_{i}^{r}\right) \quad\left(i=1, \ldots, N_{a}\right) .
$$

Step 3. Calculate maximal ABDT difference of any two wheel axles, that is, $\max _{\forall i_{1} \neq i_{2}}\left|\bar{T}_{i_{1}}-\bar{T}_{i_{2}}\right|\left(i_{1}=1, \ldots, N_{a}\right.$ and $i_{2}=$ $\left.1, \ldots, N_{a}\right)$. If this maximum is within a proper scope, the braking coordination of articulated vehicle is satisfactory. Otherwise, the braking coordination performance is poor. Obviously, the higher this maximum is, the worse the braking coordination performance becomes.

\section{Field Tests and Results}

To verify the performance of the proposed dynamic measurement system for evaluating the braking coordination, field tests were conducted on an articulated vehicle. It consists of a FOTON AUMAN $6 \times 4$ tractor, pulling a CIMC 3-axle semitrailer. That is, it totally had 6 wheel axles $\left(N_{a}=6\right)$. Moreover, it was equipped with a Novatel FLEXPAK-V2-RT20 DGPS with $20 \mathrm{~Hz}$ update rate and a ZEMIC L9L-0.1-100 kg$\mathrm{J}$ brake pedal force sensor sampled at $100 \mathrm{~Hz}$ as well as our designed WVUs with $100 \mathrm{~Hz}$ sample rate which totally had 12 modules and were installed on all 6 wheel axles. The sensor accuracies $(1 \sigma)$ are $0.01 \mathrm{~m} / \mathrm{s}$ for DGPS velocity and 1 newton

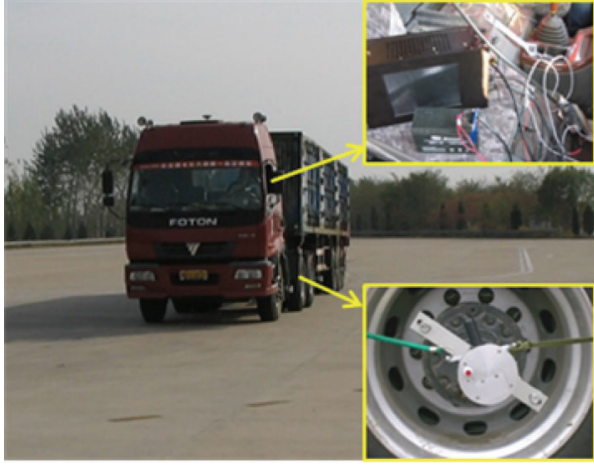

Figure 5: Articulated vehicle and test platform.

(N) for brake pedal force. The articulated vehicle and test platform are shown in Figure 5.

The tests were carried out in an open and flat area at the Proving Ground for Highway and Traffic, Ministry of Transport, China. During the tests, the tractor-semitrailer first accelerated smoothly to reach a certain preset velocity and then the emergent brake maneuver was executed to evaluate its whole braking coordination. The specific testing and operating conditions satisfy the requirements of GB7258, GB/T 26778, and GB 12676 in China [8-10]. For each test, all wheel angular velocity data were processed by the algorithms developed in Section 3 and then the evaluation method of braking coordination proposed in Section 4 was carried out.

5.1. Performance of Data Processing Algorithms. Since all wheel angular velocity data have the same processing procedure, we choose the right-rear wheel of the semitrailer to demonstrate the effectiveness of processing algorithms developed in Section 3. For the right-rear wheel, Figure 6 shows the results of detecting and correcting the gross errors through traditional Grubbs method and the developed AR(3) model in one valid braking test. For the Grubbs method, the significance level $5 \%$ is chosen to detect the gross errors and the interpolation is utilized to correct them. Further, Figure 7 gives the results of denoising through the classical and proposed wavelet algorithms for the same test. Here, the proposed one refers to the improved-thresholding wavelet filtering algorithm developed in Section 3.2. For comparison, the classical wavelet algorithm also adopts "Daubechies5" as the wavelet function and four levels of decomposition, as the proposed wavelet algorithm uses. However, the classical one adopts the common soft-thresholding based on SURE rather than the improved-thresholding scheme proposed in Section 3.2 .

From Figure 6, the original wheel angular velocity data includes gross errors at the beginning of brake. Through application of AR(3) model, the gross errors can be detected and corrected effectively. In particular, the proposed $\mathrm{AR}(3)$ is suitable for both single and consecutive gross errors. However, for consecutive gross errors, traditional Grubbs method cannot provide satisfactory results.

From Figure 7, both classical and the proposed wavelet filtering algorithms have the capability of removing a great 


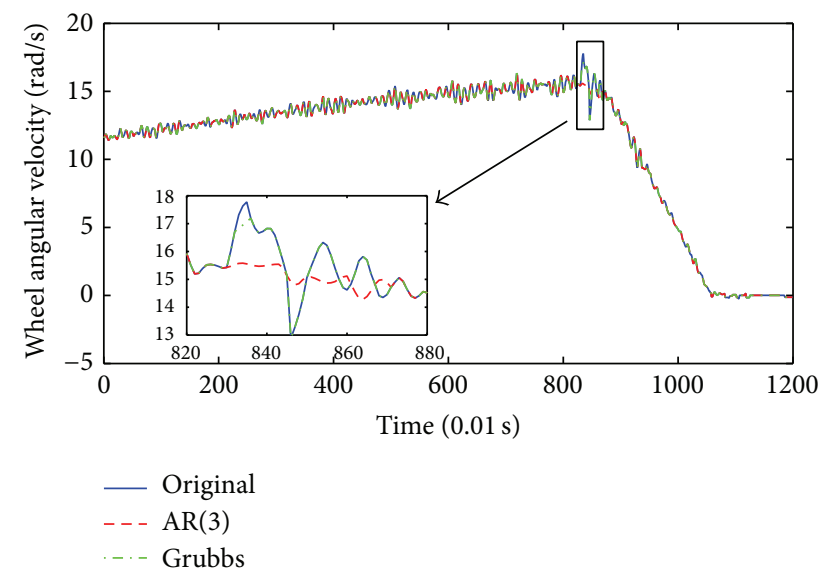

FIGURE 6: The results of detecting and correcting gross errors through Grubbs and AR(3).

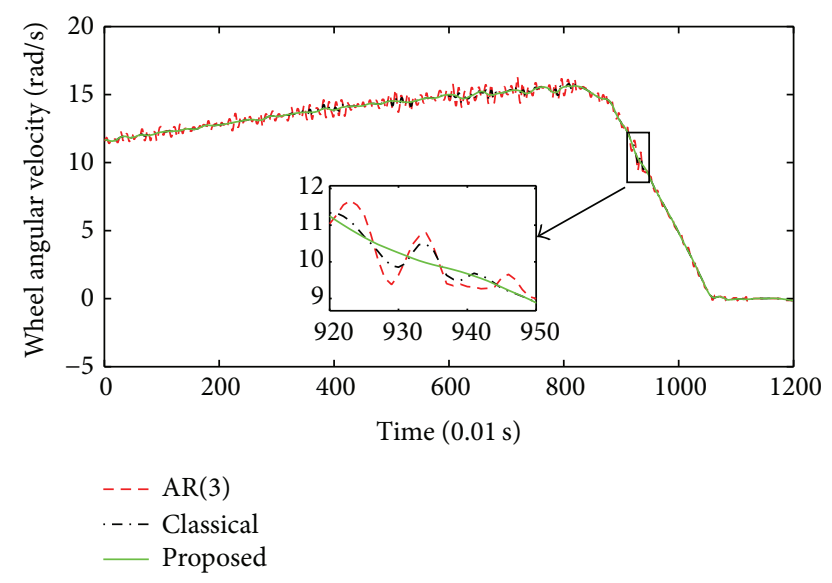

FIGURE 7: The denoising results through wavelet algorithms.

amount of noise. However, in contrast, the proposed wavelet algorithm is more obvious and effective. It can be mainly attributed to the improved-thresholding technique developed in Section 3.2, which is especially remarkable in situations of heavy noise.

\subsection{Evaluation of Braking Coordination Performance and} Discussion. When all wheel angular velocity data have been processed by the proposed AR(3) and wavelet filtering algorithms, we can evaluate the dynamic braking coordination of the whole articulated vehicle utilizing the evaluation method developed in Section 4. For the test discussed in Section 5.1, it can be found that the maximal ABDT difference occurs between the front axle of the tractor and the last axle of the semitrailer. Figure 8 shows processed velocities of four related wheels in the unified time frame, that is, left-front and rightfront wheels of the tractor and left-rear and right-rear wheels of the semitrailer. Besides, the brake pedal force is also shown in Figure 8. For clearer displaying, Figure 9 gives the local enlarged plot of Figure 8.

According to the procedures presented in Section 4, we can determine the maximal ABDT difference between

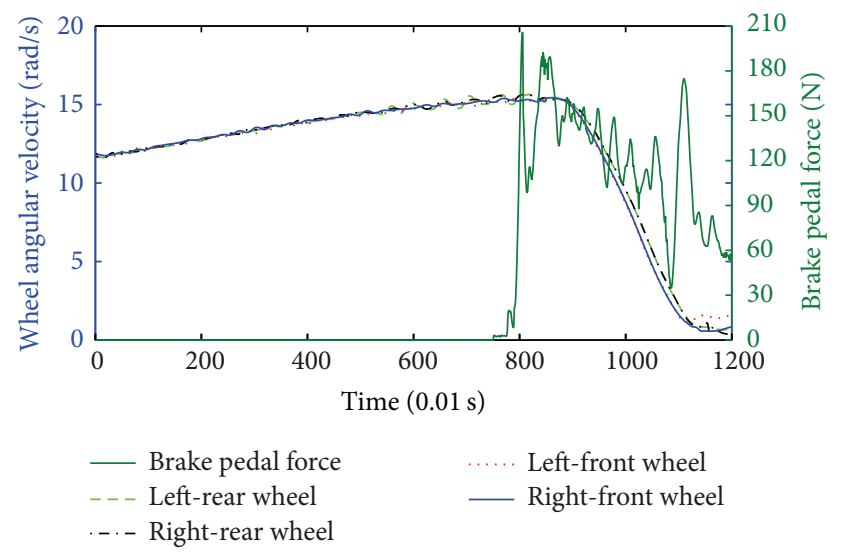

FIGURE 8: Four processed wheel velocities and brake pedal force in the unified time frame.

the front axle of the tractor and the last axle of the semitrailer; that is, $\left|\bar{T}_{1}-\bar{T}_{6}\right|=0.116 \mathrm{~s}$. Since this maximum is less than the threshold $0.2 \mathrm{~s}$ (recommended in [8-10]), it can be inferred that the braking coordination of this articulated vehicle is satisfactory in this test. Besides, according to our tests, we can make the following supplementary discussions.

(1) To achieve reliable evaluation, it is suitable to carry out 5-6 repeated tests in the same testing and operating conditions and then make statistical average processing to assess the final braking coordination.

(2) The accuracy and reliability of the proposed evaluation system are also verified by experimental results, which can actually be demonstrated from the following two aspects.

(a) According to the results of multiple field tests, it can be found that although the ABDT of each wheel is obtained and processed independently, there is only a slight difference (less than $0.015 \mathrm{~s}$ ) between ABDTs of any two wheels located at the same axle (i.e., left and right wheels at the same axle). Such result is consistent with the characteristics that the wheels located at the same axle have almost the same braking pipeline delay structure in this case. If the measuring accuracy about the wheel ABDT is larger than $0.015 \mathrm{~s}$, such consistent result cannot be obtained.

For instance, in the same test shown in Figure 9, it can be seen that the delay-time difference between left and right wheels at the front axle of the tractor is slight. Through computing, the difference between the ABDTs of left and right wheels at the front axle of the tractor is $\left|\bar{T}_{1}^{l}-\bar{T}_{1}^{r}\right|=0.012 \mathrm{~s}<$ $0.015 \mathrm{~s}\left(\bar{T}_{1}^{l}\right.$ and $\bar{T}_{1}^{r}$ are the ABDTs of left and right wheels at the front axle of the tractor, resp.). Similarly, the difference between the ABDTs of left and right wheels at the rear axle of the semitrailer, that is, $\left|\bar{T}_{6}^{l}-\bar{T}_{6}^{r}\right|$, is also slight and less than $0.015 \mathrm{~s}\left(\bar{T}_{6}^{l}\right.$ and $\bar{T}_{6}^{r}$ are the ABDTs of left and right wheels at the rear axle of the semitrailer, resp.).

(b) Also based on the results of multiple field tests, it can be found that the time difference between the ABDT of any wheel located at the $i_{1}$ th axle and the ABDT of left wheel located at the $i_{2}$ th axle $\left(i_{1} \neq i_{2}\right)$ is almost the same as the time difference between the ABDT of the same wheel at the $i_{1}$ th 


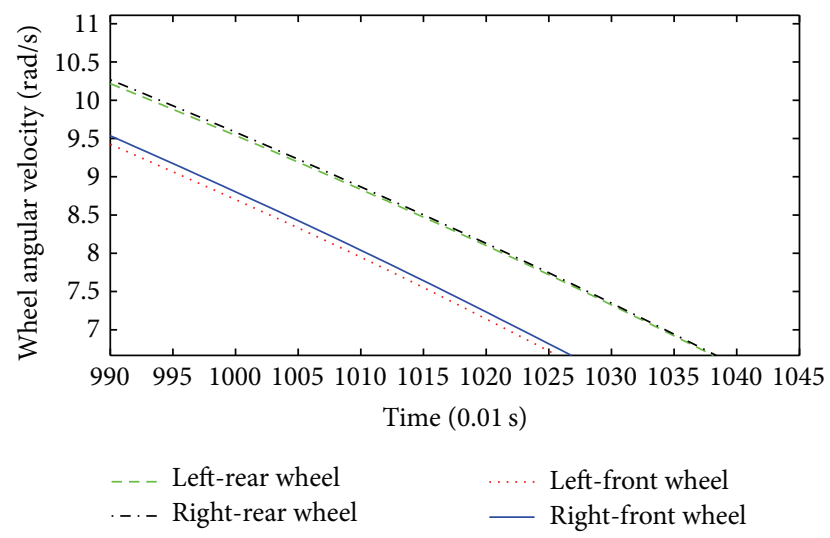

FIGURE 9: Local enlarged plot of Figure 8.

axle and the ABDT of right wheel at the $i_{2}$ th axle $\left(i_{1} \neq i_{2}\right)$. The difference between the two time differences described above is less than $0.015 \mathrm{~s}$. Obviously, if the ABDT of any wheel cannot be measured accurately, such consistent and stable result cannot be achieved. Besides, the time difference between the ABDTs of any two wheels at two different axles is obvious, which is due to different braking pipe delays they include. This obvious time difference is just the quantity we need to measure for evaluating the coordination between different axles.

As the same test shown in Figure 9, it can be found that the delay-time difference between any wheel at the front axle of the tractor and the left wheel at the rear axle of the semitrailer is almost the same as the delay-time difference between the same wheel at the front axle of the tractor and the right wheel at the rear axle of the semitrailer. Actually, through computing, we can find that both $\left\|\bar{T}_{1}^{l}-\bar{T}_{6}^{l}|-| \bar{T}_{1}^{l}-\bar{T}_{6}^{r}\right\|$ and $\| \bar{T}_{1}^{r}-$ $\bar{T}_{6}^{l}|-| \bar{T}_{1}^{r}-\bar{T}_{6}^{r}||$ are less than $0.015 \mathrm{~s}$.

\section{Conclusion}

Many severe accidents of articulated vehicles such as jackknife, snaking, and trailer-swing are caused by braking incoordination between tractor and semitrailer. To realize direct, reliable, and accurate evaluation about braking coordination, a novel dynamic measurement system is proposed in this paper.

The specially designed distributed acquisition platform is first developed to synchronously collect all wheel velocities. To obtain more accurate wheel angular velocities, the $\operatorname{AR}(3)$ model and the improved-thresholding wavelet filtering algorithm are then proposed to effectively remove gross errors and perform denoising, respectively. Further, the new evaluation method based on the differences in angular velocity dropping time of all wheels is proposed, which can directly reflect the final braking coordination of different wheels from the view of kinematics. Through field tests, it is validated that the proposed system has the capability of evaluating the braking coordination between tractor and semitrailer reliably and accurately.

\section{Competing Interests}

The authors declare that they have no competing interests.

\section{Acknowledgments}

This work was supported in part by the Science and Technology Major Project of the Ministry of Transportation of China (Grant no. 2011318223450), the National Natural Science Foundation of China (Grant no. 61273236), the Jiangsu Provincial Basic Research Program (Natural Science Foundation, Grant no. BK2010239), and the Scientific Research Foundation of Graduate School of Southeast University (no. YBJJ1637).

\section{References}

[1] L.-K. Chen and Y.-A. Shieh, "Jackknife prevention for articulated vehicles using model reference adaptive control," Proceedings of the Institution of Mechanical Engineers, Part D: Journal of Automobile Engineering, vol. 225, no. 1, pp. 28-42, 2011.

[2] M. F. J. van de Molengraft-Luijten, I. J. M. Besselink, R. M. A. F. Verschuren, and H. Nijmeijer, "Analysis of the lateral dynamic behaviour of articulated commercial vehicles," Vehicle System Dynamics, vol. 50, supplement 1, pp. 169-189, 2012.

[3] J. Woodrooffe, D. Blower, T. Gordon, P. E. Green, B. Liu, and P. Sweatman, "Safety benefits of stability control systems for tractor-semitrailers," Tech. Rep., US Department of Transportation, Washington, DC, USA, 2009.

[4] J. I. Roh, H. Lee, and W. Chung, "Control of a car with a trailer using the driver assistance system," in Proceedings of the IEEE International Conference on Robotics and Biomimetics (ROBIO '11), pp. 2890-2895, Phuket, Thailand, December 2011.

[5] M.-W. Suh, Y.-K. Park, and S.-J. Kwon, "Braking performance simulation for a tractor-semitrailer vehicle with an air brake system," Proceedings of the Institution of Mechanical Engineers, Part D: Journal of Automobile Engineering, vol. 216, no. 1, pp. 43-54, 2002.

[6] Federal motor vehicle safety standards and regulations, http:// www.nhtsa.gov/cars/rules/import/FMVSS/.

[7] "Uniform provisions concerning the approval of vehicles of categories $\mathrm{m}, \mathrm{n}$ and o with regard to braking," ECERegulation 13, 2007.

[8] China standard: GB 7258 "Safety specifications for powerdriven vehicles operating on roads", 2012.

[9] China standard: GB/T 26778 "Combination of vehicle performance requirements and test method", 2011.

[10] China standard: GB 12676 "Technical requirements and testing methods for commercial vehicle and trailer braking systems", 2014.

[11] M. Bouteldja, A. Koita, V. Dolcemascolo, and J. C. Cadiou, "Prediction and detection of jackknifing problems for tractor semitrailer," in Proceedings of the IEEE Vehicle Power and Propulsion Conference (VPPC '06), pp. 1-6, Windsor, UK, September 2006.

[12] L. K. Chen and J. Y. Hsu, "Investigation of jack-knife prevention in an articulated scaled vehicle," Vehicle System Dynamics, vol. 46, no. 1, pp. 765-777, 2008.

[13] F. P. Bayan, A. D. Cornetto III, A. Dunn, and E. Sauer, "Brake timing measurements for a tractor-semitrailer under emergency braking," SAE International Journal of Commercial Vehicles, vol. 2, no. 2, pp. 245-255, 2010. 
[14] W. D. Bartlett, "Calculation of heavy truck deceleration based on air pressure rise-time and brake adjustment," SAE Technical Papers 2632, 2004.

[15] A. L. Dunn, C. B. Tanner, D. R. Morr et al., "The influence of disablement of various brakes on the dry stopping performance and stability of a tractor-semitrailer," SAE International Journal of Commercial Vehicles, vol. 2, no. 1, pp. 1-16, 2009.

[16] S. Sun, C. Zhang, and G. Yu, "A Bayesian network approach to traffic flow forecasting," IEEE Transactions on Intelligent Transportation Systems, vol. 7, no. 1, pp. 124-132, 2006.

[17] A. Abadi, T. Rajabioun, and P. A. Ioannou, "Traffic flow prediction for road transportation networks with limited traffic data," IEEE Transactions on Intelligent Transportation Systems, vol. 16, no. 2, pp. 653-662, 2015.

[18] J. Georgy, A. Noureldin, M. J. Korenberg, and M. M. Bayoumi, "Modeling the stochastic drift of a MEMS-based gyroscope in gyro/odometer/GPS integrated navigation," IEEE Transactions on Intelligent Transportation Systems, vol. 11, no. 4, pp. 856-872, 2010.

[19] B. Liu, X. Jia, Z. Huang, and H. Li, "An improved system for 3D individualized modeling of the artificial femoral head," Visual Computer, vol. 29, no. 12, pp. 1259-1267, 2013.

[20] Y. Shi, Y. Luo, W. Zhao, C. Shang, Y. Wang, and Y. Chen, "A radiosonde using a humidity sensor array with a platinum resistance heater and multi-sensor data fusion," Sensors, vol. 13, no. 7, pp. 8977-8996, 2013.

[21] V. Béziat, J. Traherne, J.-A. Malmberg et al., "Tracing dynamic expansion of human NK-cell subsets by high-resolution analysis of KIR repertoires and cellular differentiation," European Journal of Immunology, vol. 44, no. 7, pp. 2192-2196, 2014.

[22] Z. N. Qu and J. L. Xie, "Long-term periodicity variations of the solar radius," Astrophysical Journal, vol. 762, no. 1, article 23, 2013.

[23] M. Tamazin, A. Noureldin, and M. J. Korenberg, "Robust modeling of low-cost MEMS sensor errors in mobile devices using fast orthogonal search," Journal of Sensors, vol. 2013, Article ID 101820, 8 pages, 2013.

[24] S. Dunne and B. Ghosh, "Weather adaptive traffic prediction using neurowavelet models," IEEE Transactions on Intelligent Transportation Systems, vol. 14, no. 1, pp. 370-379, 2013.

[25] O. M. Bouzid, G. Y. Tian, J. Neasham, and B. Sharif, "Envelope and wavelet transform for sound localisation at low sampling rates in wireless sensor networks," Journal of Sensors, vol. 2012, Article ID 680383, 9 pages, 2012.

[26] M. Molodova, Z. Li, A. Nunez, and R. Dollevoet, "Automatic detection of squats in railway infrastructure," IEEE Transactions on Intelligent Transportation Systems, vol. 15, no. 5, pp. 19801990, 2014.

[27] D. L. Donoho and I. M. Johnstone, "Ideal spatial adaptation by wavelet shrinkage," Biometrika, vol. 81, no. 3, pp. 425-455, 1994.

[28] T. Zhang and X. Xu, "A new method of seamless land navigation for GPS/INS integrated system," Measurement, vol. 45, no. 4, pp. 691-701, 2012. 


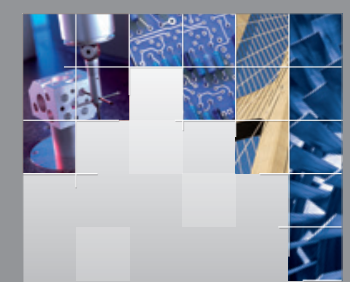

\section{Enfincering}
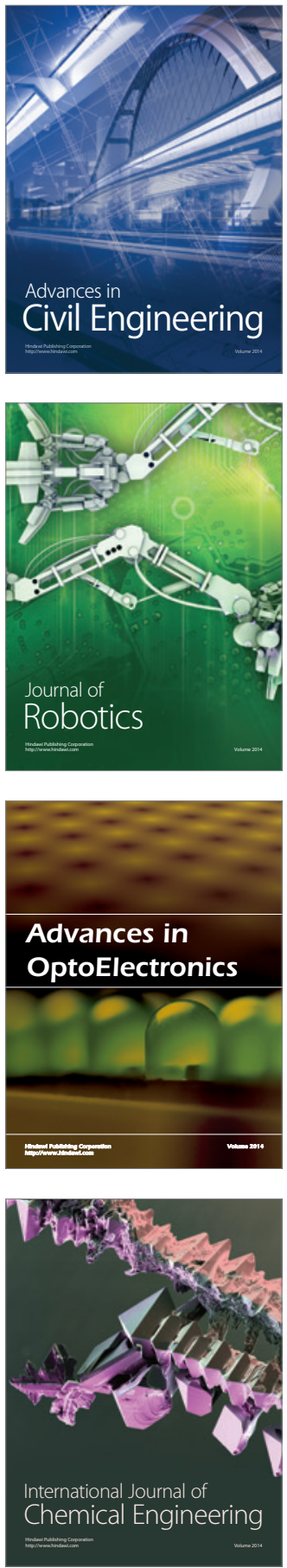

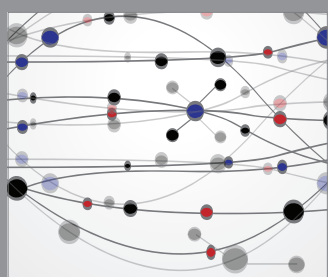

The Scientific World Journal

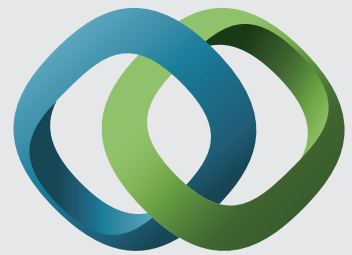

\section{Hindawi}

Submit your manuscripts at

http://www.hindawi.com
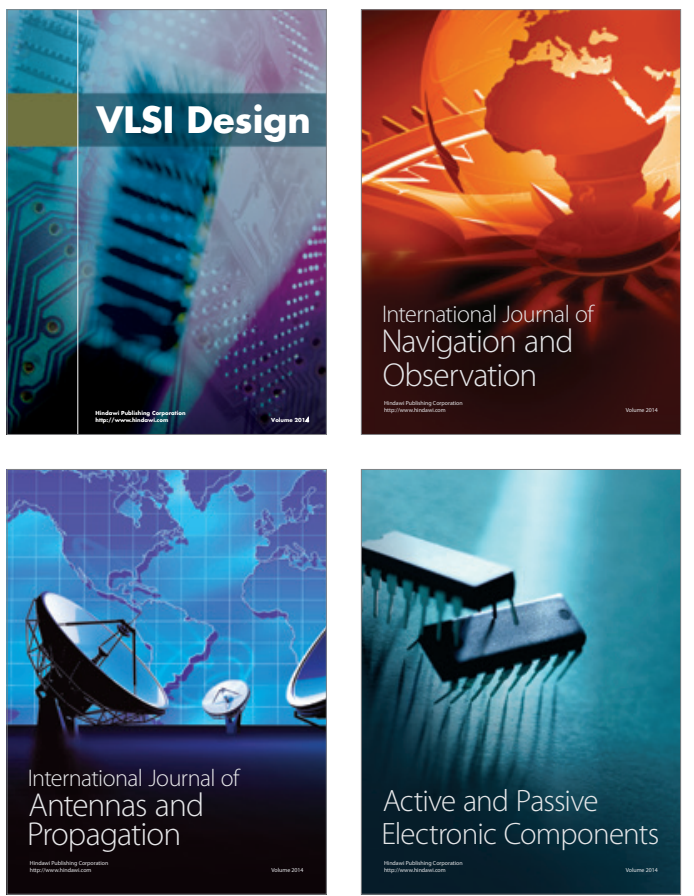
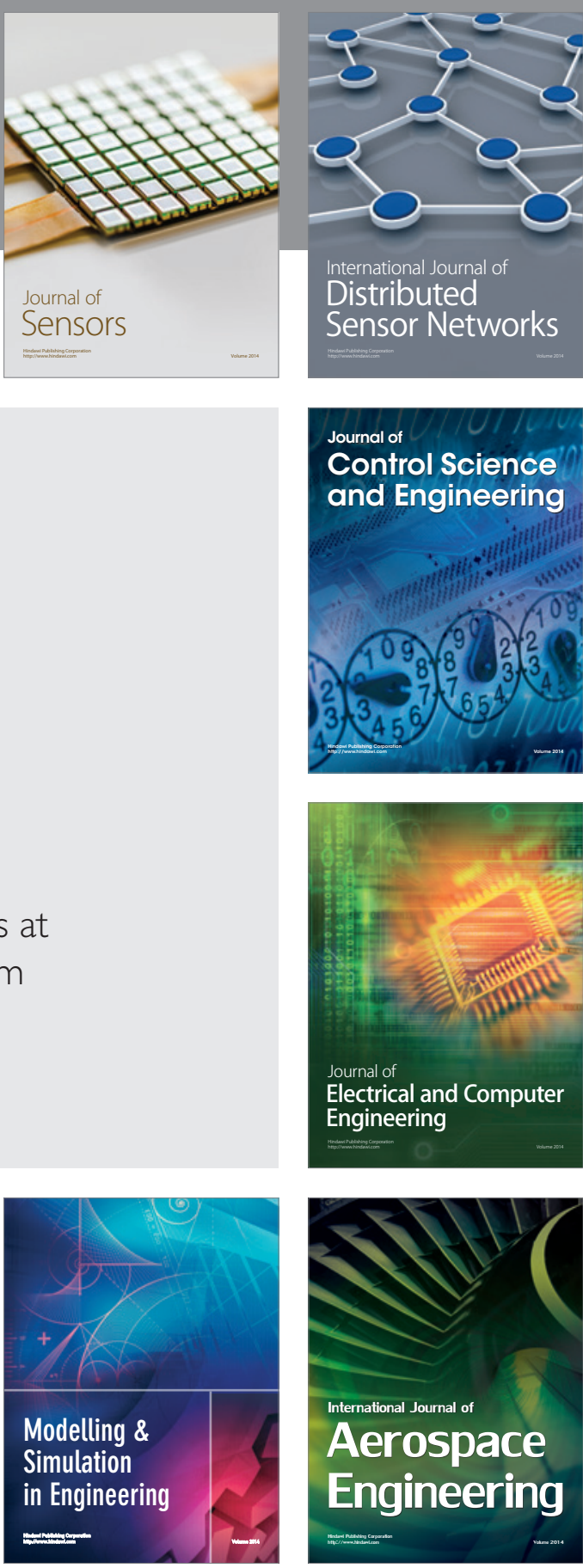

International Journal of

Distributed

Sensor Networks

Journal of

Control Science

and Engineering
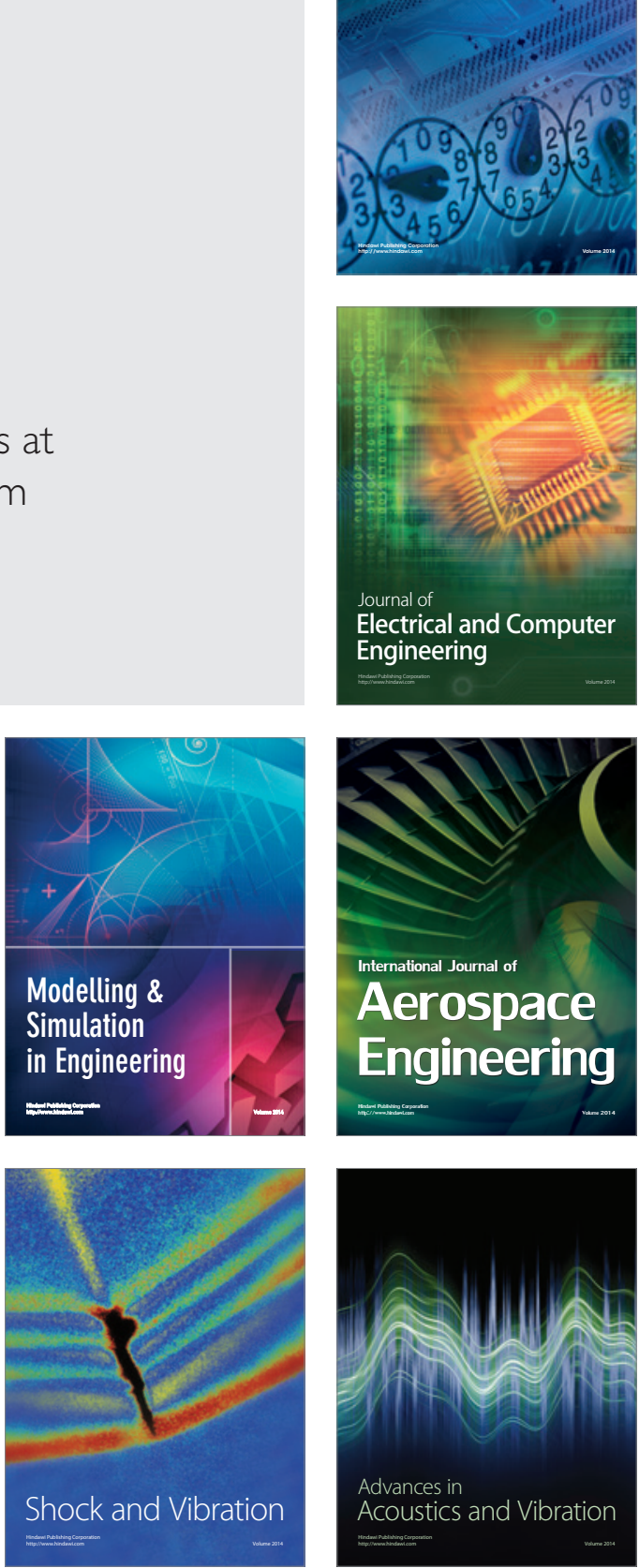